\title{
Accuracy of the equilibrium structure of sulphur dioxide*
}

\author{
Jean Demaison and Jacques Liévin \\ Spectroscopy, Quantum Chemistry and Atmospheric Remote Sensing (SQUARES), CP160/09, Faculté des Sciences, Université Libre de Bruxelles \\ (U.L.B.) Brussels, Belgium
}

\begin{abstract}
The ground state rotational spectra of 12 different isotopologues of sulphur dioxide have been refitted in a consistent way and an accurate experimental cubic force field has been determined. The experimental, semi-experimental and $a b$ initio equilibrium structures have been calculated and found in good agreement across themselves. Different approximations limiting the accuracy are discussed. In particular, the influence of centrifugal distortion is analysed. A method is proposed to estimate the higher-order rovibrational corrections ( $\gamma$-constants). Although an accurate semiexperimental structure was obtained for 19 isotopologues, it was not possible to determine and account for the breakdown of the Born-Oppenheimer approximation. Finally, it was shown that the $a b$ initio CCSDTQ level of theory was required to obtain a good agreement with the experimental structure.
\end{abstract}

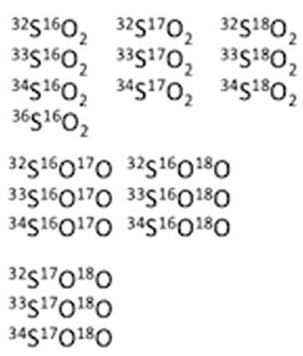

\section{ARTICLE HISTORY}

Received 4 May 2021

Accepted 23 June 2021

\section{Introduction}

Assuming that the errors are random, it is possible to use the law of propagation of errors to estimate the accuracy of an equilibrium structure. In many cases, this method concludes that the structure is highly accurate.

As it turns out, however, in the particular case of a structure determination, systematic errors are dominant. First, there is the Born-Oppenheimer approximation. Although it is a very good approximation, it limits the accuracy of the structure of a polyatomic molecule. Then, there is the way the rotation-vibration interaction constants ( $\alpha$ - and $\gamma$-constants) are obtained. Quite often, they are sensitive to anharmonic resonances and/or Coriolis interactions. The accuracy - or neglect of higher-order rotation-vibration interaction constants
( $\gamma$-constants) also affects the accuracy. Finally, the electronic correction and the centrifugal distortion correction are often neglected although, in some cases, they may have a sizable effect [1-3].

For the sake of completeness, it has also to be remembered that the accuracy of the final structure also used to depend on the accuracy of the Planck constant and of the atomic masses. However, these constants are now highly accurate and the effect of their uncertainty is negligible.

The goal of this paper is to analyse in detail these different approximations, which limit the accuracy of an equilibrium structure in the particular case of sulphur dioxide, $\mathrm{SO}_{2}$.

The reasons for this choice are numerous. It is a small triatomic molecule, whose rotational and rovibrational

CONTACT Jean Demaison jean.demaison@gmail.com Spectroscopy, Quantum Chemistry and Atmospheric Remote Sensing (SQUARES), CP160/09, Faculté des Sciences, Université Libre de Bruxelles (U.L.B.), Ave. Roosevelt, 50, B-1050 Brussels, Belgium

*This work is dedicated to Dr Jean-Marie FLAUD on the occasion of his 75th birthday.

(6) Supplemental data for this article can be accessed here. https://doi.org/10.1080/00268976.2021.1950857 
spectra have been analysed in great detail [4-6]. The ground state rotational spectra of 19 isotopologues are indeed available.

In the next section, the determination of equilibrium rotational constants is discussed. In the following section, the experimental equilibrium structures of $\mathrm{SO}_{2}$ are reviewed. Then, an experimental cubic force field of high accuracy is calculated. It allows us to determine an accurate semi-experimental equilibrium structure for 19 isotopologues of sulphur dioxide. After that, a simple method permitting to estimate the contribution of higher-order rovibrational corrections is proposed. The breakdown of the Born-Oppenheimer approximation is investigated. Finally, the $a b$ initio Born-Oppenheimer structure is calculated at the coupled-cluster level and compared to the experimental values.

\section{Determination of equilibrium rotational constants}

\subsection{Rovibrational correction [1-3, 7]}

The rotational constants $X_{v}$ for a given vibrational state $v$ of an asymmetric top are given by

$$
\begin{aligned}
X_{v}= & X_{\mathrm{e}}-\sum_{i} \alpha_{i}^{X}\left(v_{i}+\frac{1}{2}\right) \\
& +\sum_{i \geq j} \gamma_{i j}^{X}\left(v_{i}+\frac{1}{2}\right)\left(v_{j}+\frac{1}{2}\right)+\cdots
\end{aligned}
$$

where $X=A, B$ and $C$. The summations are over all normal vibrations, each characterised by the quantum number $v$. The parameters $\alpha_{i}^{X}$ and $\gamma_{i j}^{X}$ are the vibration-rotation constants and $X_{\mathrm{e}}$ are the equilibrium rotational constants, which are required to determine the equilibrium structure. They may be obtained experimentally by a least-squares fit to Equation (1) using the rotational constants of the ground state and of the excited vibrational states (fundamental, combinations and overtones) as input data. As the excited states are often perturbed by Coriolis interactions and/or anharmonic resonances, it is required to properly treat these interactions. On the other hand, the ground state rotational constants can easily be obtained experimentally, while the $\alpha_{i}^{X}$ constants are easily derived from an $a b$ initio anharmonic force field (up to cubic terms). Unfortunately, higherorder anharmonic force constants are required for calculating the $\gamma$-constants, but there is no theoretical relation so far. For this reason, the effect of the $\gamma$-constants on the structure will be analysed below.

\subsection{Centrifugal distortion correction $[2,8]$}

The experimental rotational constants are slightly different from the rigid rotor constants and a small correction due to the centrifugal distortion has to be added. The fitted rotational constants in the asymmetric reduction $(A)$ of Watson [8] (for historical reasons, it is usually preferred for $\mathrm{SO}_{2}$ ) have to be corrected using the following relations

$$
\begin{aligned}
& A=A^{A}+2 \Delta_{J}+\frac{1}{2}\left(\tau_{b b c c}+\tau_{a b a b}\right) \\
& B=B^{A}+2 \Delta_{J}+\Delta_{J K}-2 \delta_{J}-2 \delta_{K}+\frac{1}{2}\left(\tau_{a c a c}+\tau_{a b a b}\right) \\
& C=C^{A}+2 \Delta_{J}+\Delta_{J K}+2 \delta_{J}+2 \delta_{K}+\frac{1}{2} \tau_{a a b b}+\frac{1}{4} \tau_{a b a b}
\end{aligned}
$$

where $A, B$ and $C$ are the unperturbed rotational constants (after centrifugal distortion correction), whereas $A^{A}, B^{A}$ and $C^{A}$ are the experimental constants resulting from the fit using the $A$-reduction. The $\tau_{\alpha \beta \gamma \delta}$ constants are the quartic centrifugal distortion constants of Wilson and Howard [9]. They can be approximately determined using the planarity relations [10]. As their vibrational dependence, which may account for more than $10 \%$, is neglected, this correction is not expected to be accurate.

Although it is small, this correction has to be taken into account when very accurate results are desired, and the effect of its neglect will be examined.

Unfortunately, there are additional terms to Equation (2). Watson [11] has shown that there is a massdependent contribution to the potential energy. This contribution has the effect of displacing the equilibrium configuration of a particular isotope slightly from the isotopic invariant configuration at the minimum of the potential. This shift of origin will show up in the values of the rotational constants whose equilibrium values are

$$
X^{\prime}=X-\frac{1}{8}\left(\tau_{x x x x}+\tau_{x x y y}+\tau_{x x z z}\right)
$$

This correction can be important for light molecules but there are still additional terms whose detailed form has not been discussed so far. For this reason, this last correction, Equation (3), is generally neglected. However, its influence will be estimated in the present work.

\subsection{Electronic correction $[1,2,12]$}

Atomic masses are used to calculate the structure although the centre of mass of the electrons of a bonded atom often does not coincide with the position of its nucleus because the distribution of the electronic clouds 
Table 1. Experimental values for the rotation-vibration interaction constants (in $\mathrm{MHz}$ ) of ${ }^{32} \mathrm{~S}^{16} \mathrm{O}_{2}$.

\begin{tabular}{|c|c|c|c|c|c|c|}
\hline \multirow[b]{2}{*}{$X$} & \multicolumn{3}{|c|}{ Flaud and Lafferty [17] } & \multicolumn{3}{|c|}{ Morino and Tanimoto [18] } \\
\hline & $A$ & $B$ & $C$ & $A$ & $B$ & $C$ \\
\hline$\alpha_{1}$ & $-33.33(45)$ & $50.023(90)$ & $42.328(91)$ & $-36.41(75)$ & $49.51(12)$ & $41.77(10)$ \\
\hline$\alpha_{2}$ & $-1130.36(55)$ & $-2.624(33)$ & $15.685(47)$ & $-1126.68(48)$ & $-2.727(72)$ & $15.864(80)$ \\
\hline$\alpha_{3}$ & $616.57(23)$ & $34.954(39)$ & $32.405(46)$ & $613.40(62)$ & $34.11(10)$ & $31.812(81)$ \\
\hline$\gamma_{11}$ & $-0.60(22)$ & $0.049(43)$ & $0.104(44)$ & $-2.19(39)$ & $0.126(60)$ & $0.121(51)$ \\
\hline$\gamma_{12}$ & $6.86(28)$ & $0.087(55)$ & $0.404(56)$ & $-1.81(80)$ & $-0.049(64)$ & $0.512(57)$ \\
\hline$\gamma_{13}$ & $-6.25(40)$ & $0.137(73)$ & $-0.851(80)$ & $-0.33(38)$ & $0.44(55)$ & $-0.588(44)$ \\
\hline$\gamma_{22}$ & $24.77(38)$ & $-0.1728(59)$ & $-0.0791(59)$ & $28.83(23)$ & $-0.137(21)$ & $-0.002(35)$ \\
\hline$\gamma_{23}$ & $-17.85(40)$ & & $-0.422(80)$ & $-18.44(31)$ & $-0.103(40)$ & $-0.485(37)$ \\
\hline$\gamma_{33}$ & $3.88(16)$ & $-0.0351(31)$ & $0.0767(31)$ & $1.59(28)$ & $0.148(43)$ & $0.267(36)$ \\
\hline$\gamma_{222}$ & $0.571(82)$ & & & & & \\
\hline$\sum \alpha / 2^{a}$ & $-273.57(45)$ & $41.177(52)$ & $45.209(56)$ & $-274.84(54)$ & $40.447(86)$ & $44.725(76)$ \\
\hline$\sum \gamma / 4^{a}$ & $2.70(19)$ & $0.0161(25)$ & $-0.192(33)$ & $1.91(27)$ & $0.105(30)$ & $-0.044(27)$ \\
\hline$\gamma_{222} / 8$ & $0.071(10)$ & & & & & \\
\hline$X_{\mathrm{e}}-X_{0}{ }^{\mathrm{a}}$ & $-276.20(49)$ & $41.160(58)$ & $45.401(65)$ & $-276.75(61)$ & $40.342(91)$ & $44.769(81)$ \\
\hline$x_{0}$ & $60778.5526(39)$ & $10318.07332(60)$ & 8799.70375(54) & & & \\
\hline \multirow[t]{2}{*}{$X_{\mathrm{e}}$} & $60502.21(26)$ & $10359.234(38)$ & $8845.105(40)$ & $60501.77(40)$ & $10358.329(62)$ & $8844.617(47)$ \\
\hline & \multicolumn{3}{|c|}{$r_{\mathrm{e}}=1.430782(15) \AA ; \angle(O S O)=119.3297(30)^{\circ}$} & \multicolumn{3}{|c|}{$r_{\mathrm{e}}=1.4308302(17) \AA \AA ;($ OSO $)=119.33042(29)^{\circ}$} \\
\hline
\end{tabular}

${ }^{a}$ The uncertainties are calculated using the law of propagation of errors and neglecting the correlations.

around the atoms in a molecule is non-spherical. For this reason, the rotational constants have to be corrected for a small electronic effect. The corrected values of the rotational constants are given by the relation

$$
X_{\mathrm{corr}}=\frac{X_{\exp }}{1+\left(m / M_{\mathrm{P}}\right) g_{X X}}
$$

where $g_{X X}$ is unitless, $m$ and $M_{\mathrm{P}}$ being the electron and proton masses, respectively.

This last correction, although small, is not negligible in the particular case of $\mathrm{SO}_{2}$.

For the ${ }^{32} \mathrm{~S}^{16} \mathrm{O}_{2}$ isotopologue, the experimental $g_{X X}$ values are used: $g_{a a}=-0.6043(3) ; g_{b b}=-0.11634(12)$; and $g_{c c}=-0.08865(10)$ [13]. It is then possible to calculate them for other isotopologues using the electric dipole moment, $\mu=1.633 \mathrm{D}$ [14] and the shift of the centre of mass upon isotopic substitution [15].

\section{Experimental equilibrium structures of $\mathrm{SO}_{2}$}

\subsection{Isotopologues ${ }^{32} \mathrm{~S}^{16} \mathrm{O}_{2}$ and ${ }^{34} \mathrm{~S}^{16} \mathrm{O}_{2}$}

The experimental equilibrium structure of ${ }^{32} \mathrm{~S}^{16} \mathrm{O}_{2}$ has been determined many times. Very accurate values were first obtained in 1969 by Saito [16]: $r_{\mathrm{e}}=1.43076(13) \AA$ and $\angle(\mathrm{OSO})=119.33(1)^{\circ}$. More recently, the structure was redetermined using the rotational constants of many more vibrational levels by Flaud and Lafferty [17] and by Morino and Tanimoto [18]. Their results are compared in Table 1. There is a fair agreement between both studies although there are some discrepancies. In particular, the $\gamma$-constants are not compatible. The contribution of these $\gamma$-terms to the $A$-rotational constant is about $2 \mathrm{MHz}$ and, comparing the two studies, an uncertainty as large as $1 \mathrm{MHz}$ seems to be realistic. On the other hand, the contribution of the $\gamma$-terms to the $B$ - and $C$-rotational constants is small.

The ground state inertial defect is $\Delta_{0}=0.1363 \mathrm{u}^{2}$ whereas the equilibrium value is $\Delta_{\mathrm{e}}=-0.0003(8) \mathrm{u} \AA^{2}$. The uncertainty mainly comes from the rovibrational correction, which means that the equilibrium value $\Delta_{\mathrm{e}}$ is not significantly different from zero.

The structure of ${ }^{34} \mathrm{~S}^{16} \mathrm{O}_{2}$ was also accurately determined by Lafferty et al. [19].

The structure of these two isotopologues was recalculated estimating the effect of the different corrections and using more recent ground state rotational constants [20] as well as more accurate $g$-values [13]. Furthermore, the centrifugal distortion correction was calculated in a more consistent way. The rotational constants and the different corrections are given in Table 2. The centrifugal distortion correction, Equation (2) is quite small but, for the correction to the $C$-constant, there is a difference of $25 \%$ between the experimental value derived from the experimental centrifugal distortion constants and the value calculated from the harmonic force field [21]. The correction due to the mass-dependent contribution to the potential energy, Equation (3), is large for $A(1.18 \mathrm{MHz})$ but almost negligible for $B$ and $C$.

As there are only two structural parameters and three equilibrium rotational constants, the structure can be obtained in three different ways. The results are given in Table 3. The uncertainties are calculated using the law of propagation of errors, in other words, the systematic errors are not included and the true uncertainty is probably significantly larger.

It seems that the structure derived from the $I_{a}$ and $I_{b}$ moments of inertia is the most precise one. However, it does not take into account the large uncertainty of about $1 \mathrm{MHz}$ on the $\gamma$-contribution. However, despite 
Table 2. Experimental ground state and equilibrium rotational constants of sulphur dioxide with their different corrections.

\begin{tabular}{|c|c|c|c|c|c|c|}
\hline 16.32 .16 & & $A / \mathrm{MHz}$ & $B / \mathrm{MHz}$ & $\mathrm{C} / \mathrm{MHz}$ & $\Delta / u \AA^{2}$ & Ref. \\
\hline$X_{0} \exp$ & & $60778.54978(29)$ & $10318.073534(51)$ & $8799.703401(45)$ & 0.1363 & 20 \\
\hline$X_{\mathrm{e}}-X_{0}$ total $^{\mathrm{a}}$ & Equation (1) & $-276.198(489)$ & $41.160(58)$ & $45.401(65)$ & -0.0018 & 17 \\
\hline$\sum \gamma / 4$ & & $2.70(19)$ & $0.0161(25)$ & $-0.192(33)$ & & \\
\hline$g$-corr. & Equation (4) & 19.914 & 0.656 & 0.427 & 0.0012 & \\
\hline cd exp. & Equation (2) & -0.023 & -0.136 & 0.146 & -0.0003 & \\
\hline cd calc. & & -0.023 & -0.128 & 0.189 & -0.0006 & \\
\hline mass dep. & Equation (3) & 1.176 & -0.045 & -0.008 & -0.0003 & \\
\hline$X_{\mathrm{e}}$ & Equations $(1,2,4)$ & 60522.243 & 10359.754 & 8845.677 & & \\
\hline Xe + mass dep. & Equations $(1,2,3,4)$ & 60523.419 & 10359.710 & 8845.670 & & \\
\hline \multicolumn{7}{|l|}{ 16.34.16 } \\
\hline$X_{0} \exp$ & & 58991.18295(51) & 10318.50993(9) & $8761.302481(97)$ & 0.1381 & 19 \\
\hline$X_{\mathrm{e}}-X_{0}$ total $^{\mathrm{a}}$ & Equation (1) & $-267.04(254)$ & $40.7265(61)$ & $44.660(94)$ & -0.0008 & \\
\hline$\sum \gamma / 4$ & & $2.275(69)$ & $0.0071(15)$ & $-0.234(31)$ & & \\
\hline$g$-corr. & Equation (4) & 18.809 & 0.656 & 0.424 & 0.0022 & \\
\hline cd exp. & Equation (2) & -0.022 & -0.134 & 0.144 & 0.0007 & \\
\hline mass dep. & Equation (3) & 1.106 & -0.043 & -0.007 & 0.0007 & \\
\hline$X_{\mathrm{e}}$ & Equations $(1,2,4)$ & 58742.930 & 10359.759 & 8806.531 & & \\
\hline $\mathrm{Xe}+$ mass dep. & Equations $(1,2,3,4)$ & 58744.036 & 10359.716 & 8806.524 & & \\
\hline \multicolumn{7}{|l|}{16.32 .18} \\
\hline$x_{0} \exp$ & & 59101.17304(16) & $9724.643842(35)$ & $8331.560892(32)$ & 0.1379 & 6 \\
\hline$X_{\mathrm{e}}-X_{0}$ total $^{\mathrm{a}}$ & Equation (1) & $-261.85(16)$ & $37.966(29)$ & $42.124(27)$ & -0.0011 & \\
\hline$\sum \gamma / 4$ & & $3.427(34)$ & $-0.0801(62)$ & $-0.2070(56)$ & & \\
\hline$g$-corr. & Equation (4) & 19.117 & 0.580 & 0.387 & 0.0020 & \\
\hline cd exp. & Equation (2) & -0.021 & -0.123 & 0.132 & 0.0004 & \\
\hline mass dep. & Equation (3) & 1.111 & -0.041 & -0.008 & 0.0004 & \\
\hline$x_{\mathrm{e}}$ & Equations $(1,2,4)$ & 58861.839 & 9762.988 & 8373.996 & & \\
\hline Xe + mass dep. & Equations $(1,2,3,4)$ & 58862.950 & 9762.947 & 8373.988 & & \\
\hline
\end{tabular}

Table 3. Experimental equilibrium structure of sulfur dioxide (distances in $\AA$ and angles in degree).

\begin{tabular}{|c|c|c|c|c|c|c|}
\hline \multirow[b]{2}{*}{ Fit of parameter } & \multicolumn{2}{|c|}{$I_{a}, I_{b}$} & \multicolumn{2}{|c|}{$I_{b}, I_{c}$} & \multicolumn{2}{|c|}{$I_{c}, I_{a}$} \\
\hline & $\theta=\angle$ (OSO) & $r(\mathrm{SO})$ & $\angle(\mathrm{OSO})$ & $r(\mathrm{SO})$ & $\angle(\mathrm{OSO})$ & $r(\mathrm{SO})$ \\
\hline \multicolumn{7}{|l|}{${ }^{32} \mathrm{~S}^{16} \mathrm{O}_{2}$} \\
\hline Normal $^{\mathrm{a}}$ & $119.32902(25)$ & $1.4307885(33)$ & $119.3300(16)$ & $1.4307811(96)$ & $119.32884(32)$ & $1.4307848(46)$ \\
\hline Without $\gamma^{\mathrm{b}}$ & 119.33006 & 1.4307800 & 119.32605 & 1.4308090 & 119.33075 & 1.4307940 \\
\hline Without distortion ${ }^{c}$ & 119.32870 & 1.4307810 & 119.32496 & 1.4308090 & 119.32934 & 1.4309500 \\
\hline With $U^{\mathrm{d}}$ & 119.32961 & 1.4307870 & 119.33062 & 1.4307800 & 119.32944 & 1.4307840 \\
\hline Range & 0.00136 & 0.0000085 & 0.00566 & 0.0000290 & 0.00191 & 0.0001660 \\
\hline \multicolumn{7}{|l|}{${ }^{34} S^{16} O_{2}$} \\
\hline Normal $^{a}$ & $119.32866(21)$ & $1.4307908(42)$ & $119.3266(13)$ & $1.4308061(96)$ & $119.32903(32)$ & $1.4307987(84)$ \\
\hline Without $\gamma^{\mathrm{b}}$ & 119.32961 & 1.4307834 & 119.32203 & 1.4308388 & 119.33095 & 1.4308119 \\
\hline Without distortion ${ }^{c}$ & 119.32866 & 1.4307908 & 119.32656 & 1.4308061 & 119.32903 & 1.4307987 \\
\hline With $U^{\mathrm{d}}$ & 119.32923 & 1.4307896 & 119.32714 & 1.4308049 & 119.32960 & 1.4307975 \\
\hline Range & 0.00095 & 0.0000074 & 0.00511 & 0.0000338 & 0.00192 & 0.0000144 \\
\hline \multicolumn{7}{|l|}{${ }^{32} s^{16} 0^{18} 0$} \\
\hline Normal $^{\mathrm{a}}$ & $119.32958(10)$ & $1.4307917(16)$ & $119.33311(74)$ & $1.43076594(43)$ & $119.32900(12)$ & $1.43077920(19)$ \\
\hline Without $\gamma^{\mathrm{b}}$ & 119.32792 & 1.4307979 & 119.33210 & 1.43076742 & 119.32723 & 1.43078313 \\
\hline Without distortion ${ }^{c}$ & 119.32928 & 1.4307849 & 119.32814 & 1.43079321 & 119.32946 & 1.43078894 \\
\hline Range & 0.00166 & 0.0000130 & 0.00497 & 0.00002727 & 0.00224 & 0.00000974 \\
\hline
\end{tabular}

${ }^{\text {a }}$ All corrections included, except $U$, Equation (3), see text.

${ }^{\mathrm{b}}$ See last term of Equation (1).

'See Equation (2).

dEquation (3).

this uncertainty, the advantage of using $I_{a}$ and $I_{b}$ remains. When the mass-dependent contribution to the potential energy ( $U$-term), Equation (3), is considered, the changes are of the same order of magnitude as the precision of the parameters. Obviously, when $I_{a}$ is not used, the changes are much smaller. As this correction is quite small and as, furthermore, it is not possible to estimate the entire correction, it will be neglected in the following.
When the $\gamma$-terms are neglected, the changes are again of the same order of magnitude as the uncertainties, between $3 \sigma\left(I_{a}\right.$ and $\left.I_{b}\right)$ and $6 \sigma\left(I_{a}\right.$ and $\left.I_{c}\right)$. However, it has to be noted that the $\alpha$-constants used are the 'unperturbed' ones, not those derived from the fundamental vibrations,

$$
\alpha_{0}^{X}=X_{0}-X_{1}
$$


Table 4. Difference between $\alpha_{0}$ and $\alpha, \alpha_{0, v}^{X}-\alpha_{v}^{X}$ with $X=A, B$, $\mathrm{C}$ (in $\mathrm{MHz}$ ).

\begin{tabular}{lcrc}
\hline $\mathrm{v}$ & $\mathrm{A}$ & $\mathrm{B}$ & $\mathrm{C}$ \\
\hline 1 & 0.89 & -0.21 & 0.02 \\
2 & -44.04 & 0.30 & 0.17 \\
3 & 4.29 & 0.00 & 0.48 \\
\hline$\alpha_{0, i}=\alpha_{i}-2 \gamma_{i i}+\left(\gamma_{i j}+\gamma_{i k}\right) / 2$ with $i, j, k=1,2,3$. &
\end{tabular}

Table 5. Derivatives $\partial p / \partial X$ ( $p$ in $\AA$ or degree, $X$ in $\mathrm{MHz}$ ).

\begin{tabular}{lrrrr}
\hline$\partial p / \partial X$ & $\partial \theta / \partial X_{1}$ & $\partial \theta / \partial X_{2}$ & \multicolumn{1}{c}{$\partial r / \partial X_{1}$} & $\partial r / \partial X_{2}$ \\
\hline${ }^{32} \mathbf{S}^{\mathbf{1 6}} \mathbf{O}_{2}$ & & & & \\
$A=1 ; B=2$ & -0.00041 & 0.00241 & 0.0000030 & 0.0000514 \\
$B=1 ; C=2$ & 0.01649 & -0.01932 & -0.0000515 & 0.0001411 \\
$A=1 ; C=2$ & -0.00048 & 0.00331 & 0.0000015 & 0.0000706 \\
${ }^{34} \mathbf{S}^{\mathbf{1 6}} \mathbf{O}_{2}$ & & & & \\
$A=1 ; B=2$ & 0.00043 & -0.00241 & -0.0000031 & -0.0000514 \\
$B=1 ; C=2$ & -0.01608 & 0.01892 & 0.0000484 & -0.0001382 \\
$A=1 ; C=2$ & 0.00050 & -0.00334 & -0.0000015 & -0.0000712 \\
${ }^{32} \mathbf{S}^{16} \mathbf{O}^{\mathbf{1 8}} \mathbf{O}$ & & & & \\
$A=1 ; B=2$ & 0.00042 & -0.00256 & -0.0000031 & -0.0000546 \\
$B=1 ; C=2$ & -0.01799 & 0.02098 & 0.0000580 & -0.0001530 \\
$A=1 ; C=2$ & 0.00050 & -0.00348 & -0.0000016 & -0.0000742 \\
\hline
\end{tabular}

Indeed, the latter $\alpha_{0}$-constants are quite different from the 'unperturbed' $\alpha$-constants because they are significantly altered by the $\gamma$-terms, see Table 4 . The difference is quite large for the $\alpha^{A}$-constants, in particular for the $v=2$ state. In other words, to determine experimental unperturbed $\alpha$-constants, it is necessary to take into account the $\gamma$-terms. Obviously, this difficulty does not arise when the $\alpha$-constants are calculated from an anharmonic force field.

Finally, when the centrifugal correction, Equation (2), is neglected, there is a change of the same order of magnitude as the precision of the parameters, except when the pair $I_{b}, I_{c}$ is used, where the change is significant. It has also to be noted that it is important to take into account the centrifugal distortion in order to obtain an inertial defect as small as possible, see Table 2. Comparison of the results of Table 3 indicates that the pair $I_{b}$ and $I_{c}$ is to be avoided because it gives values, which are less precise and, worse, less accurate. To understand the reasons of this behaviour, the derivatives $\partial p / \partial X$ where $p$ is either $r(\mathrm{SO})$ or $\theta(\mathrm{OSO})$ and $X=A, B, C$ were calculated. They are given in Table 5 and it appears that the derivatives are much larger when the rotational constants $B$ and $C$ are used. Furthermore, as the errors on $B$ and $C$ are of opposite sign, they are additive. For the same reason, with the pair $I_{b}$ and $I_{c}$, the errors are larger when the $\gamma$-terms are neglected, although these terms are quite small for $B$ and C.

An experimental equilibrium structure was recently determined for the isotopologue ${ }^{32} \mathrm{~S}^{16} \mathrm{O}^{18} \mathrm{O}$ [6]. It is less accurate because it was determined from a small number of experimental data. However, it is also given in Table 3 and it confirms the conclusions obtained for the other two isotopologues. In summary, it is better to use the pair $I_{a}$ and $I_{b}$ to obtain an accurate structure and, taking into account the $\gamma$-terms and the centrifugal distortion correction slightly improves the accuracy. Using the results obtained for ${ }^{32} \mathrm{~S}^{16} \mathrm{O}_{2}$ and ${ }^{34} \mathrm{~S}^{16} \mathrm{O}_{2}$, it is possible to estimate a robust estimation of the structure, which is the median of the six most accurate values of Table 3 (lines 'normal' for the ${ }^{32} \mathrm{SO}_{2}$ and ${ }^{34} \mathrm{SO}_{2}$ species). It gives: $r_{\mathrm{e}}(\mathrm{SO})=1.4307897(99) \AA$ and $\angle(\mathrm{OSO})=119.32893(27)^{\circ}$. The standard deviation is derived from the median absolute value (MAD) of the residuals. The method of the weighted mean, although less robust (because it is more sensitive to errors), gives compatible results: $r_{\mathrm{e}}(\mathrm{SO})=1.4307894(22) \AA$ and $\angle(\mathrm{OSO})=119.32884(13)^{\circ}$. It is worth noticing that with this latter method, the main difference is that the uncertainties are much smaller. This confirms that the uncertainties used for the weighting are too optimistic.

\section{Anharmonic force field of sulphur dioxide}

As an anharmonic force field is available for sulphur dioxide, [21] it is possible to determine a semiexperimental structure. However, it is wise to first check the quality of this force field.

There are several experimental determinations of the force field up to quartic terms because the experimental data concerning the $\alpha$ and $x$ (anharmonicity constants) values are abundant for this molecule and because it is one of the few simple systems for which the cubic and most of the quartic potential constants can be determined uniquely, provided all $\alpha$ and $x$ values are known [22]. There are also $a b$ initio force fields. In particular, Martin [23] computed an accurate anharmonic force field at the $\operatorname{CCSD}(\mathrm{T}) / \mathrm{cc}-\mathrm{pVQZ}+1$ level of theory. This potential was adjusted by Zúñiga et al. [21]. to improve the agreement with observed vibrational energies. Watson [24] found that this latter force field gives good results for the calculation of octic centrifugal distortion constants. It was also found to predict correctly the values of the ground state sextic constants as well as the vibrational dependence of the quartic centrifugal distortion constants [6]. There is also a high-quality ab initio potential energy surface from Huang et al. but the force constants are not available [25].

The $\alpha$-constants calculated from the force field of Zúñiga et al. [21] are compared to the experimental values in Table 6. For the $B$ and $C$ rotational constants of the parent species, the differences are quite small, about $30 \mathrm{kHz}(0.08 \%)$. On the other hand, the difference for the $A$-constant is large: about $14 \mathrm{MHz}(5 \%)$. For ${ }^{34} \mathrm{~S}^{16} \mathrm{O}_{2}$, comparable results are found, except for $C$ where the difference for $\alpha_{3}$ is as large as $214 \mathrm{kHz}$. As the structures 
Table 6. Comparison of experimental and calculated $\alpha$-constants (in $\mathrm{MHz}$ ).

\begin{tabular}{|c|c|c|c|c|c|c|c|c|c|}
\hline \multirow[b]{2}{*}{$x$} & \multicolumn{3}{|c|}{$A$} & \multicolumn{3}{|c|}{$B$} & \multicolumn{3}{|c|}{$C$} \\
\hline & Exp. & Calc. $^{a}$ & Calc. $^{b}$ & Exp. & Calc. $^{a}$ & Calc. ${ }^{b}$ & Exp. & Calc. $^{a}$ & Calc. $^{b}$ \\
\hline \multicolumn{10}{|c|}{${ }^{32} \mathrm{~S}^{16} \mathrm{O}_{2}{ }^{\mathrm{c}}$} \\
\hline$\alpha_{1}$ & $-33.33(45)$ & -25.92 & -33.95 & $50.023(90)$ & 50.412 & 49.925 & $42.328(91)$ & 42.717 & 42.260 \\
\hline$\alpha_{2}$ & $-1130.36(55)$ & -1109.49 & -1130.45 & $-2.624(33)$ & -3.549 & -2.626 & $15.685(47)$ & 15.491 & 15.681 \\
\hline$\alpha_{3}$ & $616.57(23)$ & 610.88 & 616.27 & $34.954(39)$ & 35.390 & 34.970 & $32.405(46)$ & 32.664 & 32.427 \\
\hline$X_{\mathrm{e}}-X_{0}$ & $-276.198(489)$ & -262.27 & -274.06 & $41.160(58)$ & 41.127 & 41.134 & $45.401(65)$ & 45.436 & 45.184 \\
\hline \multicolumn{10}{|c|}{${ }^{34} \mathrm{~S}^{16} \mathrm{O}_{2}{ }^{d}$} \\
\hline$\alpha_{1}$ & $-16.94(17)$ & -8.89 & -16.32 & $49.131(73)$ & 49.681 & 49.176 & $41.19(10)$ & 41.705 & 41.322 \\
\hline$\alpha_{2}$ & $-1095.34(39)$ & -1074.97 & -1095.29 & $-2.42(71)$ & -3.337 & -2.413 & $15.83(12)$ & 15.709 & 15.887 \\
\hline$\alpha_{3}$ & $582.76(24)$ & 577.86 & 583.03 & $34.76(61)$ & 35.033 & 34.618 & $31.83(85)$ & 32.252 & 32.024 \\
\hline$X_{\mathrm{e}}-X_{0}$ & $-267.04(254)$ & -253.00 & -264.29 & $40.7265(61)$ & 40.688 & 40.691 & $44.660(94)$ & 44.874 & 44.616 \\
\hline \multicolumn{10}{|c|}{${ }^{32} \mathrm{~S}^{16} \mathrm{O}^{18} \mathrm{O}^{\mathrm{e}}$} \\
\hline$\alpha_{1}$ & $-43.88(12)$ & -36.86 & -44.65 & $46.454(25)$ & 46.819 & 46.393 & $35.692(24)$ & 39.926 & 39.518 \\
\hline$\alpha_{2}$ & $-1066.14(27)$ & -1049.28 & -1069.08 & $-2.699(45)$ & -3.438 & -2.596 & $14.156(40)$ & 13.962 & 14.148 \\
\hline$\alpha_{3}$ & 593.17(11) & 588.12 & 593.11 & $32.016(26)$ & 32.670 & 32.277 & $33.985(26)$ & 30.299 & 30.071 \\
\hline$X_{\mathrm{e}}-X_{0}$ & $-258.43(15)$ & -249.01 & -260.31 & $37.886(28)$ & 38.026 & 38.037 & $41.917(26)$ & 42.094 & 41.868 \\
\hline $\begin{array}{l}{ }^{a} \text { Force fi } \\
{ }^{b} \text { Force fi } \\
{ }^{c} \text { Ref [17] } \\
{ }^{d} \text { Ref [19] } \\
{ }^{e} \text { Ref. [6]. }\end{array}$ & $\begin{array}{l}\text { f Zúñiga et al. [21] } \\
\text { f this work. }\end{array}$ & & & & & & & & \\
\hline
\end{tabular}

Table 7. Quadratic and cubic force field of sulphur dioxide.

\begin{tabular}{|c|c|c|c|c|c|c|}
\hline Parameter & Unit & .Ref. [26] & Ref. [18].- & .Ref. [21] & .Ref. [27] & This work \\
\hline$f_{r r}$ & aJ $\AA^{-2}$ & 10.410 & $10.362(9)$ & 10.29387 & $10.390(34)$ & $10.29403(34)$ \\
\hline $\mathrm{f}_{\mathrm{rr}^{\prime}}$ & aJ $\AA^{-2}$ & 0.120 & $0.016(9)$ & -0.01858 & $0.067(31)$ & $-0.01707(34)$ \\
\hline$f_{r \alpha}$ & aJ $\AA^{-1}$ & 0.563 & $0.185(10)$ & 0.24806 & $0.423(64)$ & $0.24042(54)$ \\
\hline$f_{\alpha \alpha}$ & aJ & 1.640 & $1.652(2)$ & 1.65668 & $1.6516(52)$ & $1.65667(13)$ \\
\hline$f_{r r r}$ & aJ $\AA^{-3}$ & -69.8 & $-68.80(12)$ & -70.68798 & $-72.66(43)$ & $-70.415(30)$ \\
\hline$f_{r r r^{\prime}}$ & aJ $\AA^{-3}$ & 0.5 & $-0.69(7)$ & -0.75170 & $-1.81(25)$ & $-0.537(19)$ \\
\hline$f_{r r \alpha}$ & aJ $\AA^{-2}$ & 1.1 & $-1.01(12)$ & -0.94749 & $-2.90(61)$ & $-0.8375(52)$ \\
\hline$f_{r r^{\prime} \alpha}$ & aJ $\AA^{-2}$ & 1.9 & $-0.75(2)$ & -0.68010 & $-1.488(95)$ & $-0.6954(52)$ \\
\hline$f_{r \alpha \alpha}$ & aJ $\AA^{-1}$ & 0.1 & $-1.95(4)$ & -1.9226 & $-3.248(84)$ & $-1.8690(69)$ \\
\hline$f_{\alpha \alpha \alpha}$ & aJ & -2.3 & $-2.13(1)$ & -2.09574 & $-2.688(98)$ & $-2.1959(19)$ \\
\hline
\end{tabular}

derived from the $B$ and $C$ semiexperimental rotational constants of ${ }^{32} \mathrm{~S}^{16} \mathrm{O}_{2}$ and ${ }^{34} \mathrm{~S}^{16} \mathrm{O}_{2}$ give almost identical structures, it might be due to an experimental problem.

Furthermore, there is obviously a problem with the $\alpha^{A}$-constants, see Table 6 , for instance, the experimental value of $\alpha_{1}^{A}$ is $-33.33 \mathrm{MHz}$ whereas the computed value from the force field of Zúñiga is only $-25.92 \mathrm{MHz}$. As the $\alpha$-constants only depend on the quadratic and cubic force constants, it is possible to try to find the origin of the problem.

It is now possible to determine an accurate experimental quadratic force field. There are indeed enough accurate data for the vibrational frequencies of ${ }^{32} \mathrm{~S}^{16} \mathrm{O}_{2}$ [17] and ${ }^{34} \mathrm{~S}^{16} \mathrm{O}_{2}$ [19] to derive harmonic frequencies for all vibrations. Furthermore, as the quartic force field of Zúniga et al. [21] seems to be reliable, it is also feasible to derive accurate values for the equilibrium values of the quartic centrifugal distortion constants [6]. In other words, there are 16 experimental data to determine four quadratic force constants. The experimental data are listed in Tables S1 and S2 of the Supplementary
Material and the fitted force constants are compared in Table 7 to the previous determinations. The agreement is extremely good and the discrepancies observed for the $\alpha^{A}$-constants cannot be explained by inaccuracy of the quadratic force constants.

The next step is to refine the cubic force constants (six parameters) using the accurate experimental $\alpha$-constants of ${ }^{32} \mathrm{~S}^{16} \mathrm{O}_{2}$ and ${ }^{34} \mathrm{~S}^{16} \mathrm{O}_{2}$ (18 values). The least-squares system of normal equations is extremely well-conditioned and very accurate corrections are obtained. The improved values of the cubic constants are also given in Table 7. The improvement is best visible by comparing the mean of absolute values of the residuals: for the $\alpha^{A}$-constants, it decreases from $1.87 \mathrm{MHz}$ to $0.05 \mathrm{MHz}$, for the $\alpha^{B}$-constants, the improvement is as expected smaller, from $97 \mathrm{kHz}$ to $9 \mathrm{kHz}$, and, finally, for the $\alpha^{c}$-constants, the change is still smaller from $53 \mathrm{kHz}$ to $13 \mathrm{kHz}$. This confirms that there is a problem with the $\alpha^{c}$-constants, particularly for the ${ }^{34} \mathrm{~S}^{16} \mathrm{O}_{2}$ isotopologue.

Inspection of Table 7 confirms the difficulty to obtain accurate off-diagonal constants for the force field. 


\section{Semiexperimental equilibrium structures of $\mathrm{SO}_{2}$}

The modus operandi is almost the same as for the experimental structure but with two differences. The most important one is that the $\alpha$-constants, Equation (1), are not determined experimentally but are calculated from a cubic force field. Furthermore, the $\gamma$-constants are neglected.

The main advantage of this method is that only ground state rotational constants are required, provided an anharmonic force field is available. This is quite important because it is easy to determine accurate ground state rotational constants and, indeed, the ground state rotational spectra of 19 isotopologues of sulphur dioxide have been analysed. However, in order to have comparable results, it is necessary to use rotational constants of the same quality for all isotopologues.

\subsection{Ground state rotational constants}

Accurate ground state rotational constants are available for ${ }^{32} \mathrm{~S}^{16} \mathrm{O}_{2}$ [20], ${ }^{33} \mathrm{~S}^{16} \mathrm{O}_{2},{ }^{32} \mathrm{~S}^{16} \mathrm{O}^{17} \mathrm{O}[28],{ }^{34} \mathrm{~S}^{16} \mathrm{O}_{2}$ [19], ${ }^{32} \mathrm{~S}^{16} \mathrm{O}^{18} \mathrm{O}$ and ${ }^{32} \mathrm{~S}^{18} \mathrm{O}_{2}$ [6]. For the other species, Van Riet [29] has assigned many transitions. For a complete list of references, see [4] where a full set of rotational constants is also listed. However, the results of the fits are of very different quality. For this reason, most of the spectra were reanalysed using the mixed estimation method $[30,31]$. In this method, auxiliary information is added to the data matrix for the least-squares fits. This auxiliary information, called predicate observations by us are approximate values of the quartic and sextic centrifugal distortion constants. For symmetric substitutions of atoms in $\mathrm{SO}_{2} \mathrm{C}_{2 \mathrm{v}}$ molecules, centrifugal distortion constants $P$ and $P^{\prime}$ of the ground vibrational states of the main and substituted species can be estimated using the following relation [32]

$$
P_{i j}^{\prime}=\left(\frac{A\left[\mathrm{~S}^{18} \mathrm{O}_{2}\right]}{A\left[\mathrm{~S}^{16} \mathrm{O}_{2}\right]}\right)^{i}\left(\frac{C\left[\mathrm{~S}^{18} \mathrm{O}_{2}\right]}{C\left[\mathrm{~S}^{16} \mathrm{O}_{2}\right]}\right)^{j} P_{i j}
$$

where $i$ is the power of $J_{z}^{2}$ in the Hamiltonian and $j$ the value of $(m-2 i) / 2, m$ being the total power of the corresponding term.

For the isotopologues of $C_{s}$ symmetry, it was assumed that the variations upon isotopic substitution are additive. For instance,

$$
\begin{aligned}
& P_{i j}\left[{ }^{33} S^{16} O^{18} O\right] \\
& \quad=P_{i j}\left[{ }^{32} S^{16} O^{18} O\right]+P_{i j}\left[{ }^{33} S^{16} O_{2}\right]-P_{i j}\left[{ }^{32} S^{16} O_{2}\right]
\end{aligned}
$$

This approximation gives satisfactory results in most cases because the rotation of the principal axes upon isotopic substitution is quite small.

The choice of the associated uncertainty for the weighting of the predicates is important: $10 \%$ of the value of the parameter gave satisfactory results. Furthermore, an extensive residual analysis [30] was performed to check the weighting and to identify possible misassignments. In most cases, the sextic constants $\Phi_{J K}$ and $\phi_{J K}$ have a negligible contribution to the frequencies. For this reason, they were kept fixed at the predicted values. However, as these predicted values are derived from experimental values, which are rather inaccurate, they have to be considered as fitting parameter without physical value. The rotational parameters are listed in Table S3 of the Supplementary Material.

\subsection{Structure from the improved force field}

First, it is useful to look at the $I_{b}^{\mathrm{SE}}$ moments of inertia of the $C_{2 v}$ isotopologues because it allows us to check the accuracy of the force field. Indeed,

$$
I_{b}^{\mathrm{e}}=2 m(\mathrm{O}) r^{2} \sin ^{2} \frac{\theta}{2}
$$

Thus, dividing $I_{b}^{\mathrm{SE}}$ by the mass of oxygen permits to obtain $r \sin (\theta / 2)$, which is isotopically invariant. The results are shown in Table $S 4$ of the Supplementary Material. The median value is $r \sin (\theta / 2)=1.23489232(11) \AA$. It is extremely accurate and the invariance is well verified. As expected, the largest deviations are found for the isotopologues with nuclear quadrupole hyperfine structure because their ground state rotational constants are one order of magnitude less accurate. The range at $10^{-5} \AA$ is 10 times larger than the standard deviation but, if the isotopologues with nuclear quadrupole hyperfine structure (NQHFS) are eliminated $\left({ }^{33} \mathrm{~S}\right.$ and $\left.{ }^{17} \mathrm{O}\right)$, it decreases to $2 \times 10^{-6} \AA$.

In principle, it is also possible to use the $I_{a}^{\mathrm{SE}}$ moments of inertia to derive $r \cos (\theta / 2)$ which should be invariant upon isotopic substitution but the situation is more complicated because the $\gamma$-terms are not negligible. This point will be discussed in Section 6.

The structure was determined from the moments of inertia $I_{a}$ and $I_{b}$ in the same way as in Section 3 using the improved force field of Section 4 . The result for each isotopologue is given in Table S5 of the Supplementary Material and their median values in Table 8 . The standard deviation is calculated from the MAD. It is a robust estimator but it does not take into account the systematic errors, which are probably not negligible. However, it is possible to estimate their effect. From the comparison with the experimental values, it is possible to assign 
Table 8. Semiexperimental structure (SE) of sulphur dioxide (bond length in $\AA$ and angle in degrees).

\begin{tabular}{|c|c|c|c|c|c|c|c|}
\hline \multirow[b]{2}{*}{ Force field } & \multirow[b]{2}{*}{ Used data } & \multicolumn{3}{|c|}{$\theta_{\mathrm{e}}=\angle(\mathrm{OSO})$} & \multicolumn{3}{|c|}{$r_{\mathrm{e}}(\mathrm{SO})$} \\
\hline & & Median & mean & exp - se & median & mean & $\exp -$ se \\
\hline Exp. & & $119.32893(27)$ & & & $1.4307897(99)$ & & \\
\hline This work & $I_{a}, I_{b}$ & $119.329872(81)$ & 119.329665 & 0.00094 & $1.4307858(15)$ & 1.4307879 & -0.0000039 \\
\hline This work + scaling ${ }^{a}$ & $I_{a}, I_{b}$ & $119.328783(55)$ & 119.328740 & -0.00015 & $1.43079396(93)$ & 1.4307947 & 0.0000043 \\
\hline This work + scaling ${ }^{a}$ & $I_{b}, I_{c}$ & $119.331264(23)$ & 119.330900 & 0.00233 & $1.4307767(14)$ & 1.4307777 & -0.0000130 \\
\hline Ref[21]. + scaling ${ }^{a}$ & $I_{a}, I_{b}$ & $119.328811(40)$ & 119.328771 & -0.00012 & $1.4307943(10)$ & 1.4307950 & 0.0000047 \\
\hline
\end{tabular}

${ }^{a}$ The scaling was used to estimate the contribution of the $\gamma$-terms, see text

Table 9. Experimental and calculated rovibrational corrections $A_{\mathrm{e}}-A_{0}(\mathrm{MHz})$ for several isotopologues of sulphur dioxide.

\begin{tabular}{lllll}
\hline Isotopologue & 16.32 .16 & 16.34 .16 & 16.32 .18 & $18.34 .18^{\mathrm{a}}$ \\
\hline From the force field $^{\mathrm{b}}$ & -262.3 & -253.0 & -249.0 & -227.6 \\
Force field $^{\mathrm{b}}+$ scaling $^{\mathrm{c}}$ & -276.2 & -266.4 & -262.2 & -239.7 \\
Improved force field $^{\mathrm{d}}$ & -274.6 & -264.3 & -260.3 & -237.9 \\
Experimental value $^{\mathrm{e}}$ & -276.2 & -267.0 & -261.9 & \\
From $^{\mathrm{f}} \mathrm{f}_{\mathrm{e}}$ & -276.5 & -266.4 & -262.8 & -239.7 \\
From $_{\mathrm{e}}^{\mathrm{SEg}}$ & -277.1 & -267.0 & -263.4 & -240.3 \\
\hline
\end{tabular}

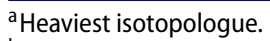

${ }^{b}$ Ref. (21).

'Scaling factor $=276.198 / 262.265=1.053$.

d This work, see text.

eSee Table 2.

${ }^{\mathrm{f}}$ Derived from the ground state rotational constant and the experimental equilibrium structure.

${ }^{g}$ Derived from the ground state rotational constant and the semiexperimental equilibrium structure.

an error of about $2 \mathrm{MHz}$ to $A_{\mathrm{e}}-A_{0}$ (mainly due to the neglect of the $\gamma$-terms) and less than $0.1 \mathrm{MHz}$ for $B_{\mathrm{e}}-B_{0}$, see Tables 6 and 9. It gives an error of $0.0009^{\circ}$ for the angle and $0.000008 \AA$ for the bond length. It is almost one order of magnitude larger than the standard deviation derived from the MAD. Another way to estimate the accuracy is to make a comparison with the experimental structure. For the angle, the difference is $\theta^{\mathrm{SE}}-\theta^{\mathrm{EXP}}=0.0009^{\circ}$ and, for the bond length, it is $r^{\mathrm{SE}}-r^{\mathrm{EXP}}=-0.000004$ $\AA$. These values are in agreement with the preceding estimation.

The main weak point of this method is that the $\gamma$ terms are neglected whereas they are not negligible for the $A$-constant. However, this problem can be remedied by scaling the force field value $\Delta A=A_{\mathrm{e}}-A_{0}$. As the range of $\Delta A$ is not large, about $35 \mathrm{MHz}$, it is possible to scale the force field values using the experimental value of the parent species, which is probably the most accurate one. In other words, the force field values of $\Delta A$ are multiplied by $276.198 / 274.06$ (experimental value/computed value). The result for each isotopologue is also given in Table S5 of the Supplementary Material and their median values in Table 8 . The standard deviation of the parameters is indeed slightly smaller and the comparison with the experimental values better: $\theta^{\mathrm{SE}}-\theta^{\mathrm{EXP}}=-0.00015^{\circ}$ for $\angle(\mathrm{OSO})$ and, for the bond length, it is $r^{\mathrm{SE}}-r^{\mathrm{EXP}}=0.000004 \AA$ for the bond length.

\subsection{Structure from the force field of Zúñiga et al. [21]}

The case of sulphur dioxide is extremely favourable because there is enough accurate experimental data permitting to calculate a reliable force field up to cubic terms. For this reason, it is interesting to investigate the possibility to determine a semiexperimental structure from the existing force field of Zúñiga et al. [21].

The main difficulty is the large systematic error for $A_{\mathrm{e}}-A_{0}$, which makes difficult to determine an accurate structure using the $A$ and $B$ rotational constants because it would induce a systematic error of about $0.006^{\circ}$ on the $\angle(\mathrm{OSO})$ angle and $4 \times 10^{-4} \AA$ on the SO bond length. Using instead $B$ and $C$ reduces significantly the systematic error: less than $0.00014^{\circ}$ for the angle and less than $4 \times 10^{-5} \AA$ for the bond length. Compared to the uncertainty of the experimental structure, $0.00027^{\circ}$ for the angle and $10^{-5} \AA$ for the bond length, using $B$ and $C$ seems acceptable although not perfect. The detailed results are given in Table S5 of the Supplementary Material and their median values in Table 8. The standard deviation of the parameters is quite small but the comparison with the experimental values less favourable: $\theta^{\mathrm{SE}}-\theta^{\mathrm{EXP}}=0.00233^{\circ}$ and, for the bond length, it is $r^{\mathrm{SE}}-r^{\mathrm{EXP}}=-0.000013 \AA$. For the $\angle(\mathrm{OSO})$ angle, it is almost ten times the standard deviation of the experimental value.

Fortunately, it is still possible to calculate the semiexperimental structure using the $A$ and $B$ rotational constants. The force field value of $\Delta A=A_{\mathrm{e}}-A_{0}$ is only $5 \%$ of the experimental value (in absolute value). As the range of $\Delta A$ is not large, it is possible to scale the force field values as done in Section 5.2. In other words, the force field values of $\Delta A$ are multiplied by $\left(\sum \alpha / 2\right)_{\exp } /\left(\sum \alpha / 2\right)_{\text {forcefield }}=276.198 / 262.265$. To check that this method does not induce errors larger than one $\mathrm{MHz}$, the scaled values are compared to the experimental values and to the values derived from the best experimental structure in Table 9. The agreement is indeed satisfactory and allows us to determine a structure, which is likely to be accurate as it is confirmed by the results. The detailed results are given in Table $\mathrm{S} 5$ of the Supplementary Material and their median values in Table 
8. The standard deviation of the parameters is quite small and, more interestingly, the comparison with the experimental values quite favourable: $\theta^{\mathrm{SE}}-\theta^{\mathrm{EXP}}=-0.00012^{\circ}$ and, for the bond length, it is $r^{\mathrm{SE}}-r^{\mathrm{EXP}}=-0.000005 \AA$. In other words, the accuracy is comparable to that found with the improved force field based on a scaling of $\Delta A$, although the method is much simpler.

\section{Estimation of the $\gamma$-parameters}

It is tempting to try to obtain $r \cos (\theta / 2)$ from $I_{a}^{\mathrm{SE}}$ because

$$
I_{a}^{\mathrm{e}}=2 \frac{m(\mathrm{O}) m(\mathrm{~S})}{M} r^{2} \cos ^{2} \frac{\theta}{2}
$$

where $M$ is the total mass of the molecule. Unfortunately, the result is not constant as it should be but increases with the mass of the molecule. This is mainly due to the neglect of the $\gamma$-constants in the calculation of $I_{a}^{\mathrm{SE}}$.

The higher-order rovibrational constants, $\gamma$, Equation (1), are not negligible for molecules with large rotational constants as $\mathrm{SO}_{2}$. However, their determination requires the rotational constants of many vibrationally excited states, most of them being perturbed. As a consequence, these $\gamma$-constants are often inaccurate, one of the few exceptions being ${ }^{32} \mathrm{~S}^{16} \mathrm{O}_{2}$ and ${ }^{34} \mathrm{~S}^{16} \mathrm{O}_{2}$. It is their contribution to the moment of inertia, $I_{\mathrm{e}}$, which is interesting. It may be written

$$
I_{e}(a, g)=I_{e}(a)-\delta(g)
$$

where $I_{\mathrm{e}}(\alpha, \gamma)$ is the equilibrium moment of inertia calculated taking into account the $\alpha$ - and $\gamma$-constants whereas $I_{\mathrm{e}}(\alpha)$ is the equilibrium moment of inertia calculated by only taking into account the $\alpha$-constants, and

$$
\delta(\gamma)=\frac{h}{8 \pi^{2}} \frac{\sum \gamma / 4}{B_{\mathrm{e}}^{2}(\alpha, \gamma)}
$$

For $\mathrm{S}^{16} \mathrm{O}_{2}$ it gives (in $\mathrm{u} \AA^{2}$ ) $0.000373(27)$ for the ${ }^{32} \mathrm{~S}$ species and $0.000333(1)$ for the ${ }^{34} \mathrm{~S}$ species. These values are close. Furthermore, it has to be noted that all the $\gamma$-constants are assumed to be free of perturbation, which is unlikely. Actually, for a diatomic molecule, using the expression of the Dunham constant $Y_{21}=\gamma$ [33], it is possible to show that $\delta(\gamma)$ is invariant upon isotopic substitution. We may assume that it remains a valid approximation for the $C_{2 v}$ isotopologues of $\mathrm{SO}_{2}$ for which there is no rotation of the principal axis system upon isotopic substitution. This assumption may be checked by calculating $I_{a}(r)$ from the best equilibrium structure and comparing it to the semi-experimental value $I_{a}^{\mathrm{SE}}$. The results are shown in Table S6 of the Supplementary Material, where the less accurate species with quadrupole hyperfine structure have been dropped.
Taking into account the uncertainties, it may be concluded that the $\delta(\gamma)$ value may be assumed to be constant upon isotopic substitution with a mean value of $\delta(\gamma)=0.000402(13) \mathrm{u}^{2}$. This value is slightly larger than the experimental values but the standard deviation does not take into account the systematic errors.

There is a simpler way to determine $\delta(\gamma)$ by using the least-squares method where the input data are the $I_{a}^{\mathrm{SE}}$ and the parameters to be determined are $\delta(\gamma)$ and $r \cos (\theta / 2)$. The results are: $\delta(\gamma)=0.00066(18) \mathrm{u}^{2}$ and $r \cos (\theta / 2)=0.7226533(76) \AA$. These results are not expected to be very accurate because the number of data is quite small, six. However, taking into account the small number of degrees of freedom, the value of $\delta(\gamma)$ is compatible with the previous determinations. Furthermore, using the value of $r \sin (\theta / 2)$ determined in Section 5.2, it allows to obtain an equilibrium structure in good agreement with the previous determinations: $r=1.4307994(55) \AA$ and $\theta=119.32805(28)^{\circ}$.

It is tempting to try to calculate the structure by using the differences of moments of inertia. The differences eliminate the contribution of $\delta(\gamma)$ as well as the systematic errors. However, this method has to be used with caution because the difference of two close values is an unstable operation. This is one of the main shortcomings of the substitution method[34, 35].

\section{7. $A \boldsymbol{b}$ initio equilibrium structure}

There was some debate concerning the structure of $\mathrm{SO}_{2}$ when compared to $\mathrm{O}_{3}$, the latter molecule having a rather large biradical character. This was solved by Takeshita et al. [36] who showed that $\mathrm{SO}_{2}$ has a closed shell nature with a double-bonded structure. Thus, the structure optimisation of $\mathrm{SO}_{2}$ should be easier than that of $\mathrm{O}_{3}$.

The coupled-cluster theory including single and double excitations (CCSD) [37] augmented with a perturbational estimate of the effects of connected triple excitations, $\operatorname{CCSD}(\mathrm{T})$ [38], with a basis set of quadruple zeta quality (cc-pwCVQZ or aug-cc-pwCVQZ for polar bonds) [39] is known to give satisfactory results. For a collection of molecules containing second-row atoms, it was found that the bond lengths are too large by 0.00024 $\AA$ (mean value) with a standard deviation of only 0.00097 $\AA$ [40]. This good result is mainly due to a compensation of errors because the introduction of connected quadruples (CCSDTQ) increases the bond length by $0.001-0.002$ $\AA$ whereas the basis set extension from quadruple zeta to sextuple zeta shortens it by about the same amount. Thus, although the convergence of the geometry is not fully achieved at the quadruple zeta level, using a larger basis set should not improve much the result. 
Table 10. Born-Oppenheimer equilibrium structure of $\mathrm{SO}_{2}$ (distances in $\AA$ and angles in degree).

\begin{tabular}{|c|c|c|c|}
\hline Method & Basis set & $r(\mathrm{SO})$ & $\theta=\angle(\mathrm{OSO})$ \\
\hline$\overline{r_{\mathrm{e}}(\exp )^{\mathrm{a}}}$ & & $1.4307897(99)$ & $119.32893(27)$ \\
\hline$r_{\mathrm{e}}(\mathrm{SE})^{\mathrm{b}}$ & & $1.43079396(93)$ & $119.328783(55)$ \\
\hline CCSD(T)_AE & aug-cc-pwCVTZ & 1.43613993 & 119.35052 \\
\hline $\operatorname{CCSD}(\mathrm{T}) \_\mathrm{AE}$ & aug-cc-pwCVQZ & 1.43109531 & 119.38922 \\
\hline $\operatorname{CCSD}(T) \_A E$ & aug-cc-pwCV5Z & 1.42957226 & 119.36741 \\
\hline $\operatorname{CCSD}(\mathrm{T}) \mathrm{AE}$ & aug-cc-pwCV6Za & 1.42786 & 119.440 \\
\hline $\operatorname{CCSD}(\mathrm{T}) \_\mathrm{AE}$ & aug-cc-pwCV $\infty Z$ & 1.42797 & 119.344 \\
\hline $\operatorname{CCSD}(\mathrm{T})-\mathrm{F} 12 \mathrm{AE}^{\mathrm{C}}$ & cc-pCVQZ-F12 & 1.42977 & 119.3618 \\
\hline $\operatorname{CCSD}(\mathrm{T})-\mathrm{F} 12 \mathrm{FC}$ & aug-cc-pV5Z-F12d & 1.42958 & 119.3567 \\
\hline CCSDTQ & e & 1.4305 & 119.40 \\
\hline MRCI-F12 ${ }^{\mathrm{d}}$ & auq-cc-pV5Z & 1.42964 & 119.3968 \\
\hline Extrapolation ${ }^{f}$ & & 1.43087716 & 119.32333 \\
\hline
\end{tabular}

${ }^{\mathrm{a} C o r r e c t i o n ~ V 5 Z ~} \rightarrow$ V6Z calculated with the MP2/aug-cc-pVnZ level of theory.

${ }^{\mathrm{b}}$ See Table S5.

${ }^{\mathrm{C}}$ The $\operatorname{CCSD}(\mathrm{T})-\mathrm{F} 12 \mathrm{~b}$ approach has been used with a scaling of the triples.

${ }^{d}$ After correction for the core correlation, see Table S8.

'See Table S7.

fSee text.

The structure was optimised at the $\operatorname{CCSD}(\mathrm{T})$ level of theory by means of the Molpro programme [41, 42], all electrons being correlated (AE) with the aug-ccpwCVQZ and aug-cc-pwCV5Z basis sets. The results are given in Table 10. Although the convergence is not completely achieved at the aug-cc-pwCVQZ level, the bond length is only $0.0003 \AA$ too large, and, as anticipated, the results are more accurate with this later basis set than with the larger aug-cc-pwCV5Z for which the bond length is $0.0012 \AA$ too short. Actually, the basis set convergence is not yet achieved at the aug-cc-pwCV5Z level. Furthermore, the rather large value of the coupled-cluster $T_{1}$ diagnostic [43] at 0.022 (CCSD/cc-pVTZ value) indicates that the possible existence of some non-dynamical correlation explaining the slow convergence of the $\operatorname{CCSD}(\mathrm{T})$ method, this implies that it would be necessary to go beyond $\operatorname{CCSD}(\mathrm{T})$.

First, the $\operatorname{CCSD}(\mathrm{T})$ structure was estimated at infinite basis size by extrapolating the aug-cc-pwCVQZ and augcc-pwCV5Z results with the following empirical equation [44]

$$
\Delta r(\mathrm{CBS})=\frac{n^{3} r(n)-(n-1)^{3} r(n-1)}{n^{3}-(n-1)^{3}}-r(n-1)
$$

where $n$ indicates the cardinal number of the larger basis set. This extrapolation formula is known to have an accuracy of about $0.001 \AA$ when only first-row atoms are involved, at least for covalent bonds. In the case of $\mathrm{SO}_{2}$, the accuracy of the extrapolation could be worse. To confirm the correct order of magnitude of this correction, the structure was also optimised at the $\operatorname{CCSD}(\mathrm{T})$ level of theory with the aug-cc-pV5Z and aug-cc-pV6Z basis sets. The correction (aug-cc-pV5Z $\rightarrow$ aug-cc-pV6Z) is
$\Delta r=-0.00171 \AA$ and $\Delta \theta=+0.073^{\circ}$. These corrections confirm the validity of the extrapolation.

One difficulty with the $\operatorname{CCSD}(\mathrm{T})$ method is its slow convergence. To obviate it, we exploited the much faster convergence of the explicitly correlated $\operatorname{CCSD}(\mathrm{T})-\mathrm{F} 12$ method [45], used with the cc-pVnZ-F12 [46] and cc-pCVnZ-F12 [47] basis sets series, specifically optimised for the F12 approaches. Indeed, when going from cc-pVQZ-F12 to cc-pV5Z-F12, the decrease in bond length and in bond angle is of $0.0002 \AA$ and $0.004^{\circ}$, respectively, with the $\operatorname{CCSD}(\mathrm{T})$-F12 method, whereas it is of $0.0015 \AA$ and $0.022^{\circ}$, respectively, with the standard $\operatorname{CCSD}(\mathrm{T})$ method. However, it does not solve the problem of the non-dynamical correlation, the bond length being too short after core correction, see Table S7 of the Supplementary Material. We first compared the standard aug-cc-p-VnZ and cc-pVnZ-F12 basis sets, see Table S7 of the Supplementary Material. It indicates that, at the V5Z level, the difference is almost negligible: +0.00018 $\AA$ for the bond length and $-0.0054^{\circ}$ for the bond angle. However, it is known that combining F12 methods with basis sets not specifically optimised for them may lead to erratic convergence [48]. For this reason, we also used the recently developed aug-cc-pVnZ-F12 basis sets of Sylvetsky et al. [49], which includes $d$ to $h$ diffuse functions in addition to cc-pVnZ-F12. We adopted in our calculations their recommendations for the values of the geminal exponent and for the choice of the resolution of identity (RI) complementary auxiliary basis set. The results are also given in Table S7 of the Supplementary Material. They show that the additional diffuse functions do not act significantly on the geometry. Including the core correction, the (aug)-cc-pV5Z-F12 and cc-pCVQZF12 results are almost identical, taking into account the fact that the extension of the basis set from VQZ to V5Z leads to a tiny shortening of $0.00021 \AA$ for the bond and of $0.0039^{\circ}$ for the angle. These results are better than with the CCSD(T)_AE/aug-cc-pwCVnZ values, which are too small for $n \geq 4$, see Table 10 . However, the bond length is still slightly too short by $0.0002 \AA$ compared to the experimental values.

It remains the problem of the non-dynamical correlation. To investigate it, the structure was first optimised with the CFOUR programme [50] at the CCSD, CCSD (T), CCSDT and CCSDTQ [51] levels of theory. Because the CCSDTQ calculation is extremely time consuming, small basis sets were used: cc-pVDZ, DZP, TZP [52], and def-SVP [53]. It is fortunately known that the small correction $\mathrm{CCSD}(\mathrm{T}) \rightarrow$ CCSDTQ can be estimated with such small basis sets [54].

These calculations confirm the lengthening of the bond length of about $0.0025 \AA$ and the shortening of the bond angle of about $0.09^{\circ}$ when going from $\operatorname{CCSD}(\mathrm{T})$ to 
CCSDTQ. The detailed results are given in Table S8 of the Supplementary Material and the final results in Table 10.

To do away with this difficulty, instead of using the CCSDTQ method, we carried out calculations with the multireference configuration interaction method (MRCI) [55] and, more specifically, the MRCI-F12 method [56, 57] implemented in Molpro. In these calculations, the contribution of unlinked clusters was taken into account by means of the Davidson correction for relaxed reference [58]. The detailed results are also given in Table S7 of the Supplementary Material and the final result in Table 10. As shown in Table 10, the MRCI value is in perfect agreement with the $\operatorname{CCSD}(\mathrm{T})-\mathrm{F} 12$ results, indicating that the non-dynamical correlation effects are probably not important.

As a further check, it might be interesting to try the extrapolation of the computed $a b$ initio rotational constants to infinite basis set limit in order to reproduce the experimental GS rotational constants. This method should minimise the effects of the slow convergence. This modus operandi was developed by Kummli et al. [59]. The structure is optimised at the triple (aug-ccpwCVTZ) and quadruple (aug-cc-pwCVQZ) zeta levels with the $\operatorname{CCSD}(\mathrm{T})$ method and the parameters are extrapolated so that the derived rotational constants are equal to the semiexperimental rotational constants. However, this method does not give satisfactory results because the distance between the aug-cc-pwCVTZ and aug-cc-pwCVQZ results is large $(0.005 \AA$ for $r)$ and the relationship between the rotational constants and the geometrical parameters is obviously not linear. In the present case, it is better to use the moments of inertia and $d_{a}=[r \cos (\theta / 2)]^{2}$ and $d_{b}=[r \sin (\theta / 2)]^{2}$ as parameters. The moments of inertia may be written

$$
I_{g}(x)=a_{g}+b_{g} \times d_{g}(x)
$$

with $g=a, b$ and $x=$ wCVTZ, wCVQZ or experimental. $a_{g}$ and $b_{g}$ are determined using $x=$ wCVTZ and wCVQZ, then, $d_{g}$ (experimental) is obtained using $I_{g}$ (experimental). This method gives good results, see Table 10 and is not sensitive to the accuracy of the rovibrational corrections $\Delta A$ and $\Delta B$ with $\partial r / \partial \Delta A=$ $-0.000003 \AA \mathrm{MHz}^{-1}, \partial r / \partial \Delta B=-0.000005 \AA \mathrm{MHz}^{-1}$, $\partial \theta / \partial \Delta A=0.00041$ degree $\mathrm{MHz}^{-1}$, and $\partial \theta / \partial \Delta B=$ -0.00024 degree $\mathrm{MHz}^{-1}$. Comparing the experimental rovibrational corrections with the $a b$ initio values computed by Martin [23], we may assume an error of $4 \%$, which implies an uncertainty of $4 \cdot 10^{-5} \AA$ for $r$ and of 0.005 degree for $\theta$. Comparison with the semiexperimental structure shows that the extrapolated values are slightly more accurate than the $\operatorname{CCSD}(\mathrm{T})$ _AE/ccpwCVnZ ones. However, this method cannot be applied to larger molecules. Interestingly, when compared to either the experimental, or semiexperimental, or extrapolated values, the CCSD $(\mathrm{T}) \_\mathrm{AE} / \mathrm{cc}-\mathrm{pwCVQZ}$ results are accurate and there is not much improvement by using either the $\operatorname{CCSD}(\mathrm{T})-\mathrm{F} 12$ or the MRCI methods.

\section{Conclusion}

The equilibrium structure of sulphur dioxide has been determined using either the experimental or the semiexperimental methods. The effect of different approximations on the accuracy has been tested and, at the end, an accurate equilibrium structure has been obtained independently for nineteen isotopologues. In particular, a simple method permitting to estimate the contribution of the $\gamma$-constants is proposed.

As the final accuracy is high, about $10^{-5} \AA$, it is possible to verify whether the Born-Oppenheimer breakdown (BOB) may be observed. The Born-Oppenheimer (BO) approximation has been studied in great detail for diatomic molecules. A typical example is $\mathrm{CO}$. The $\mathrm{CO}$ bond length calculated from the equilibrium rotational constant of ${ }^{12} \mathrm{C}^{16} \mathrm{O}$ is $1.128336 \AA$, but Watson [60] has shown that the $\mathrm{BO}$ length is $1.128243 \AA$, that is, a difference of $10^{-4} \AA$. There is no quantitative theory for polyatomic molecules. However, very accurate analyses indicate that the $\mathrm{BO}$ breakdown is also visible in linear triatomic molecules as $\mathrm{CO}_{2}$ [61]. The possibility of the $\mathrm{BO}$ breakdown has also been tested in the case of $\mathrm{HC} \equiv \mathrm{CH}[62,63]$. To point out a BOB, it is enough to check whether the geometry varies with the mass of the molecule. No such effect was observed and it can thus be concluded that the BOB is smaller than $10^{-4} \AA$.

Supplementary Material

\section{Acknowledgements}

The authors are grateful to Jean-Marie Flaud for a critical reading of the manuscript.

\section{Disclosure statement}

No potential conflict of interest was reported by the author(s).

\section{Funding}

J.L. thanks the Consortium des Équipements de Calcul Intensif (CÉCI), funded by the Fonds de la Recherche Scientifique de Belgique (F.R.S.-FNRS) under grant number 2.5020.11 and by the Walloon Region, for computational ressources.

\section{References}

[1] J. Vázquez and J. F. Stanton, in Equilibrium Molecular Structures: From Spectroscopy to Quantum Chemistry, edited by J. Demaison, J. E. Boggs and A. G. Császár (CRC Press, Boca Raton, 2011), pp. 53-87. 
[2] A. Perrin, J. Demaison, J.-M. Flaud, W. J. Lafferty, and K. Sarka, in Equilibrium Molecular Structures: From Spectroscopy to Quantum Chemistry, edited by J. Demaison, J. E. Boggs, and A. G. Császár (CRC Press, Boca Raton, 2011), pp. 89-124.

[3] H.D. Rudolph and J. Demaison, Equilibrium Molecular Structures: From Spectroscopy to Quantum Chemistry, edited by J. Demaison, J. E. Boggs, and A. G. Császár (CRC Press, Boca Raton, 2011), pp. 125-158.

[4] F.J. Lovas, J. Phys. Chem. Ref. Data. 7, 1445-1750 (1978). doi:10.1063/1.555588

[5] F.J. Lovas, J. Phys. Chem. Ref. Data. 14, 395-488 (1985). doi:10.1063/1.555729

[6] L. Margulès, R.A. Motiyenko and J. Demaison, J. Quant. Spectrosc. Radiat. Transfer. 253, 107153 (2020). doi:10.1016/j.jqsrt.2020.107153

[7] I. M. Mills, Vibration-rotation Structure in Asymmetric and Symmetric Molecules, in Molecular Spectroscopy: Modern Research, edited by K. N. Rao and C.W. Mathews (Academic Press, New York, 1972). pp. 115-140.

[8] J. K. G. Watson, (1977) Aspect of Quartic and Sextic Centrifugal Effects on Rotational Energy Levels, in Vibrational Spectra and Structure, Vol 6, edited by J. R. Durig (Elsevier, Amsterdam), pp. 2-89.

[9] E.B. Wilson and J.B. Howard, J. Chem. Phys. 4, 260-268 (1936). doi:10.1063/1.1749833

[10] K. Yamada and M. Winnewisser, Z Naturforsch. A. 31, 131-138 (1976). doi:10.1515/zna-1976-0205

[11] J.K.G. Watson, Mol. Phys. 15, 479-490 (1968). doi:10. 1080/00268976800101381

[12] W. Gordy and R.L. Cook, Microwave Molecular Spectra (Wiley, New York, 1984).

[13] A.W. Ellenbroek and A. Dymanus, Chem. Phys. Lett. 42, 303-306 (1976). doi:10.1016/0009-2614(76)80369-7

[14] D. Patel, D. Margolese and T.R. Dyke, J. Chem. Phys. 70, 2740-2747 (1979). doi:10.1063/1.437860

[15] J.M. Vacherand and J. Demaison, Z. Naturforsch. A. 41, 879-880 (1986). doi:10.1515/zna-1986-0616

[16] S. Saito, J. Mol. Spectrosc. 30, 1-16 (1969). doi:10.1016/ 0022-2852(69)90231-8

[17] J.-M. Flaud and W.J. Lafferty, J. Mol. Spectrosc. 161, 396-402 (1993). doi:10.1006/jmsp.1993.1245

[18] Y. Morino and M. Tanimoto, J. Mol. Spectrosc. 166, 310-320 (1994). doi:10.1006/jmsp.1994.1197

[19] W.J. Lafferty, J.-M. Flaud, E.H.A. Ngom and R.L. Sams, J. Mol. Spectrosc. 253, 51-54 (2009). doi:10.1016/j.jms. 2008.09.006

[20] H.S.P. Müller and S. Brünken, J. Mol. Spectrosc. 232, 213-222 (2005). doi:10.1016/j.jms.2005.04.010

[21] J. Zúñiga, A. Bastida and A. Requena, J. Chem. Phys. 115, 139-148 (2001). doi:10.1063/1.1377893

[22] K. Kuchitsu and Y. Morino, Bull. Chem. Soc. Japan. 38, 814-824 (1965). doi:10.1246/bcsj.38.814

[23] J.M.L. Martin, J. Chem. Phys. 108, 2791-2800 (1998). doi:10.1063/1.475670

[24] J.K.G. Watson, J. Mol. Struct. 795, 263-270 (2006). doi:10.1016/j.molstruc.2006.02.038

[25] X. Huang, D.W. Schwenke and T.J. Lee, J. Chem. Phys. 140, 114311 (2014). doi:10.1063/1.4868327

[26] S. Carter, I.M. Mills, J.N. Murrel and A.J.C. Varandas, Mol. Phys. 45, 1053-1066 (1982). doi:10.1080/002689782001 00801
[27] O.N. Ulenikov, E.S. Bekhtereva, S. Alanko, V.-M. Horneman, O.V. Gromova and C. Leroy, J. Mol. Spectrosc. 257, 137-156 (2009). doi:10.1016/j.jms.2009.07.005

[28] H.S.P. Müller, J. Farhoomand, E.A. Cohen, B. BrupbacherGatehouse, M. Schäfer, A. Bauder and G. Winnewisser, J. Mol. Spectrosc. 201, 1-8 (2000). doi:10.1006/jmsp.2000. 8072

[29] R. Van Riet and G. Steenbeckeliers, Ann. Soc. Sci. Bruxelles. 97, 117-153 (1983).

[30] J. Demaison, The Method of Least Squares, in: in Equilibrium Molecular Structures: From Spectroscopy to Quantum Chemistry, edited by J. Demaison, J. E. Boggs, and A. G. Császár (CRC Press, Boca Raton, 2011), pp. 29-52.

[31] D.A. Belsley, Conditioning Diagnostics. Collinearity and Weak Data in Regression (Wiley, New York, 1991).

[32] O.N. Ulenikov, E.S. Bekhtereva, Y.V. Krivchikova, Y.B. Morzhikova, T. Buttersack, C. Sydow and S. Bauerecker, J. Quant. Spectrosc. Radiat. Transfer. 166, 13-22. (2015). doi:10.1016/j.jqsrt.2015.07.004

[33] C.H. Townes and A.L. Schawlow, Microwave Spectroscopy (Dover, New York, 1975).

[34] J. Demaison and H.D. Rudolph, J. Mol. Spectrosc. 215, 78-84 (2002). doi:10.1006/jmsp.2002.8610

[35] J. Demaison, N.C. Craig, A.R. Conrad, M.J. Tubergen and H.D. Rudolph, J. Phys. Chem. A. 116, 9116-9122 (2012). doi:10.1021/jp305504x

[36] T.Y. Takeshita, B.A. Lindquist and T.H. Dunning Jr, J. Phys. Chem. A. 119, 7683-7694 (2015). doi:10.1021/acs. jpca.5b00998

[37] G.D. Purvis, I.I.I. and R and J. Bartlett, J. Chem. Phys. 76, 1910-1918 (1982). doi:10.1063/1.443164

[38] K. Raghavachari, G.W. Trucks, J.A. Pople and M. Head-Gordon, Chem. Phys. Lett. 157, 479-483 (1989). doi:10.1016/S0009-2614(89)87395-6

[39] K.A. Peterson and T.H. Dunning, J. Chem. Phys. 117, 10548-10560 (2002). doi:10.1063/1.1520138

[40] S. Coriani, D. Marchesan, J. Gauss, C. Hättig, T. Helgaker and P. Jörgensen, J. Chem. Phys. 123, 184107 (2005). doi:10.1063/1.2104387

[41] H.-J. Werner, P.J. Knowles, G. Knizia, F.R. Manby and M. Schütz., WIRES. 2, 242 (2012).

[42] H.-J. Werner, P. J. Knowles, G. Knizia, F. R. Manby, M. Schütz, P. Celani, W. Györffy, D. Kats, T. Korona, R. Lindh, A. Mitrushenkov, G. Rauhut, K. R. Shamasundar, T. B. Adler, R. D. Amos, S. J. Bennie, A. Bernhardsson, A. Berning, D. L. Cooper, M. J. O. Deegan, A. J. Dobbyn, F. Eckert, E. Goll, C. Hampel, A. Hesselmann, G. Hetzer, T. Hrenar, G. Jansen, C. Köppl, S. J. R. Lee, Y. Liu, A. W. Lloyd, Q. Ma, R. A. Mata, A. J. May, S. J. McNicholas, W. Meyer, T. F. Miller III, M. E. Mura, A. Nicklass, D. P. O’Neill, P. Palmieri, D. Peng, K. Pflüger, R. Pitzer, M. Reiher, T. Shiozaki, H. Stoll, A. J. Stone, R. Tarroni, T. Thorsteinsson, M. Wang, and M. Welborn. MOLPRO, version 2019. 2, a package of ab initio programs. see https://www.molpro.net.

[43] T. J. Lee and P. R. Taylor, Int. J. Quantum Chem. Symp. 23,199-207 (1989).

[44] C. Puzzarini, J. Phys. Chem. A. 113, 14530-14535 (2009). doi:10.1021/jp9040327

[45] T.B. Adler, G. Knizia and H.-J. Werner, J. Chem. Phys. 127, 221106 (2007). doi:10.1063/1.2817618 
[46] K.A. Peterson, T.B. Adler and H.-J. Werner, J. Chem. Phys. 128, 084102 (2008). doi:10.1063/1.2831537

[47] J.G. Hill, S. Mazumder and K.A. Peterson, J. Chem. Phys. 132, 054108 (2010). doi:10.1063/1.3308483

[48] G. Hill, K.A. Peterson, G. Knizia and H. Werner, J. Chem. Phys. 131, 194105 (2009). doi:10.1063/1.3265857

[49] N. Sylvetsky, M.K. Kesharwany and J.M.L. Martin, J. Chem. Phys. 147, 134106 (2017). doi:10.1063/1.4998332

[50] J.F. Stanton, J. Gauss, L. Cheng, M.E. Harding, D.A. Matthews, P.G. Szalay. CFOUR, Coupled-Cluster techniques for Computational Chemistry, a quantum- chemical program package, With contributions from A.A. Auer, R.J. Bartlett, U. Benedikt, C. Berger, D.E. Bernholdt, Y.J. Bomble, O. Christiansen, F. Engel, R. Faber, M. Heckert, O. Heun, M. Hilgenberg, C. Huber, T.-C. Jagau, D. Jonsson, J. Jusélius, T. Kirsch, K. Klein, W.J. Lauderdale, F. Lipparini, T. Metzroth, L.A. Mück, D.P. O’Neill, D.R. Price, E. Prochnow, C. Puzzarini, K. Ruud, F. Schiffmann, W. Schwalbach, C. Simmons, S. Stopkowicz, A. Tajti, J. Vázquez, F. Wang, J.D. Watts and the integral packages MOLECULE (J. Almlöf and P.R. Taylor), PROPS (P.R. Taylor), ABACUS (T. Helgaker, H.J. Aa. Jensen, P. Jørgensen, and J. Olsen), and ECP routines by A. V. Mitin, C. van Wüllen. For the current version, see http://www.cfour.de.

[51] M. Kállay and P.R. Surján, J. Chem. Phys. 115, 2945-2954 (2001). doi:10.1063/1.1383290
[52] A. Schäfer, H. Horn and R. Ahlrichs, J. Chem. Phys. 97, 2571-2577 (1992). doi:10.1063/1.463096

[53] F. Weigend and R. Ahlrichs, Phys. Chem. Chem. Phys. 7, 3297-3305 (2005). doi:10.1039/b508541a

[54] T.A. Ruden, T. Helgaker, P. Jørgensen and J. Olsen, J. Chem. Phys. 121, 5874-5884 (2004). doi:10.1063/1.178 0155

[55] H.-J. Werner and P.J. Knowles, J. Chem. Phys. 89, 5803-5814 (1988). doi:10.1063/1.455556

[56] T. Shiozaki, G. Knizia and H.-J. Werner, J. Chem. Phys. 134, 034113 (2011). doi:10.1063/1.3528720

[57] T. Shiozaki and H.-J. Werner, Mol. Phys. 111, 607-630 (2013). doi:10.1080/00268976.2013.779393

[58] H.-J. Werner, M. Kállay and J. Gauss, J. Chem. Phys. 128, 034305 (2008). doi:10.1063/1.2822905

[59] D.S. Kummli, H.M. Frey and S. Leutwyler, Chem. Phys. 367, 36-43 (2010). doi:10.1016/j.chemphys.2009.10.017

[60] J.K.G. Watson, J. Mol. Spectrosc. 45, 99-113 (1973). doi:10.1016/0022-2852(73)90179-3

[61] J.-L. Teffo and J.F. Ogilvie, Mol. Phys. 80, 1507-1524 (1993). doi:10.1080/00268979300103181

[62] F. Tamassia, E. Cané, L. Fusina and G. Di Lonardo, Phys. Chem. Chem. Phys. 18, 1937-1944 (2016). doi:10.1039/ C5CP05997F

[63] J. Liévin, J. Demaison, M. Herman, A. Fayt and C. Puzzarini, J. Chem. Phys. 134, 064119 (2011). doi:10.1063/ 1.3553203 